\title{
Roles of lipid metabolism in keloid development
}

\author{
Chenyu Huang ${ }^{1,2}$ and Rei Ogawa ${ }^{*^{*}}$
}

\begin{abstract}
Keloids are common cutaneous pathological scars that are characterised by the histological accumulation of fibroblasts, collagen fibres, and clinically significant invasive growth. Although increasing lines of research on keloids have revealed genetic and environmental factors that contribute to their formation, the etiology of these scars remains unclear. Several studies have suggested the involvement of lipid metabolism, from a nutritional point of view. However, the role that lipid metabolism plays in the pathogenesis and progression of keloids has not previously been reviewed. The progress that has been made in understanding the roles of the pro- and antiinflammatory lipid mediators in inflammation, and how they relate to the formation and progression of keloids, is also outlined. In particular, the possible relationships between mechanotransduction and lipid metabolites in keloids are explored. Mechanotransduction is the process by which physical forces are converted into biochemical signals that are then integrated into cellular responses. It is possible that lipid rafts and caveolae provide the location of lipid signaling and interactions between these signaling pathways and mechanotransduction. Moreover, interactions between lipid signaling pathway molecules and mechanotransduction molecules have been observed. A better understanding of the lipid profile changes and the functional roles lipid metabolism plays in keloids will help to identify target molecules for the development of novel interventions that can prevent, reduce, or even reverse pathological scar formation and/or progression.
\end{abstract}

Keywords: Lipid metabolism, Pathological scar, Keloid, Inflammation, Mechanotransduction

\section{Introduction}

Keloids are a type of cutaneous pathological scar. Regarding their clinical characteristics, they usually "invade" into the neighboring healthy skin with a leading edge that is often erythematous and pruritic, and they are difficult to treat. In terms of their pathological characteristics, they exhibit the accumulation of fibroblasts and collagen fibres that are hyalinised and eosinophilic [1]; as such, they can be considered as a fibroproliferative disorder of the skin [2]. Increasing research into the etiology of keloids has mainly centered on genetic factors [3] and local factors, such as wound tension [4], sebum secretion [5], and neurogenic inflammation [6]. However, several studies by Louw have suggested, mainly from the nutritional point of view, that essential fatty acids may be involved in the formation and progression of keloids [7-10], which has galvanised research into the contribution of nutrition in keloid

\footnotetext{
* Correspondence: r.ogawa@nms.ac.jp

${ }^{1}$ Department of Plastic, Reconstructive and Aesthetic Surgery, Nippon

Medical School, 1-1-5 Sendagi Bunkyo-ku, Tokyo 113-8603, Japan

Full list of author information is available at the end of the article
}

development and progression. However, the roles that lipid metabolism may play in keloid pathogenesis have not previously been reviewed.

Lipids mainly constitute the storage fat triglyceride and the lipoids. The lipoids include a wide range of functionally-active molecules, such as phospholipids, glycolipids, and cholesterol. In the skin, they are mainly found in biomembrane structures (e.g., phospholipids) and the stratum corneum (e.g., ceramides), where they serve as membrane constituents and provide secondary messengers. More importantly, they are functionally involved in local inflammation and intracellular signal transduction. A better understanding of the lipid profile changes and functional roles of lipid metabolism in keloids will help to identify target molecules for the development of novel interventions that can prevent, reduce, or even reverse pathological scar formation and/or progression.

\section{Constitutional changes in lipid profiles in keloid}

When comparing the mean concentrations of lipids, the ratio of triglycerides in keloidal skin is $60 \%$ of that in normal skin, although it has similar ratio of cholesterol

\section{Biomed Central}


and fatty acids. The cholesterol ester and wax ester levels are reduced to $67 \%$ and $20 \%$ of the levels in normal skin, respectively [11]. This may reflect changes in the mechanisms that cause these esters to accumulate in the skin, namely their transfer from the dermis, adipose tissue and serum, their synthesis in the epidermis, and their modification by bacterial enzymes [11,12]. In relation to this, patients with keloids have been found to consume higher levels of dietary linoleic acid (LA) and arachidonic acid (AA) than recommended (7-11 g/d), and they also consume $\alpha$-linolenic acid (ALA), eicosapentaenoic acid (EPA), and docosahexaenoic acid (DHA) at levels below the recommended 1.1-1.5 g/d [9]. Moreover, keloids bear higher levels of AA than the skin of keloid-prone and non-keloid-prone patients [10]. It may result from (1) excessive dietary intake of AA itself and its precursor LA by patients with keloids, (2) the activated phospholipase A2 $\left(\mathrm{PLA}_{2}\right)$ for AA release from the membrane pool, and (3) the infiltration of lymphocytes and cytokine secretion in the keloid edges which deplete inflammatory competitor of EPA [10]. Moreover, topical glucocorticosteroids, which are often used to treat keloids, can inhibit the synthesis in the epidermis and cultured keratinocytes of the three key stratum corneum lipids, namely cholesterol, fatty acids, and ceramides. Such epidermal lipid synthesis inhibition decreases the levels of lipid precursors for lamellar body formation and lamellar bilayer generation [13]. This observation supports the notion that changes in the lipid composition of keloids may be associated with their progression.

\section{Relationship between lipid metabolism and inflammation in keloids}

Keloids are clinically characterized by chronic inflammation of the leading edges during their invasion into the neighboring healthy skin. This is supported by the presence of infiltrating inflammatory cells on histology [2]. It has been suggested that this prolonged, active inflammatory reaction is due to cyclical skin tension, which stimulates mechanotransduction pathways [14]; it may also induce the production of neuropeptides that promote neurogenic inflammation pathways [6]. Recently, it was suggested that another etiological factor of keloids is altered lipid metabolism, particularly the metabolic processes that relate to essential fatty acids. It is possible that these alterations may promote the inflammatory reaction in keloids.

High levels of AA are a prominent characteristic of keloids [10]. AA is the source of several downstream products, including the classic eicosanoids: namely, leukotrienes (LTs), such as $\mathrm{LTB}_{4}$; prostanoids of prostaglandins (PGs), such as $\mathrm{PGE}_{2}$; prostacyclins, such as PGI; and thromboxanes (TXs) (Figure 1). These eicosanoids are generally considered to be pro-inflammatory modulators. However, AA is also the source of the nonclassic eicosanoids, called lipoxins (LXs), which serve to downregulate inflammation (Figure 1). Notably, although $\mathrm{PGE}_{2}$ is classically thought to be pro-inflammatory, it is increasingly being realized that it can act in an antiinflammatory fashion as well, as it inhibits the production of the pro-inflammatory cytokines tumor necrosis factor- $\alpha$ (TNF- $\alpha)$ and interleukin-1 $\beta$ (IL-1 $\beta)$ [15] and improves the production of the anti-inflammatory lipoxins by inducing 15-lipoxygenase (15-LOX) [16,17]. This is in accordance with the fact that, compared to normal human dermal fibroblasts, keloid-derived fibroblasts (KFs) have a diminished capacity to produce $\mathrm{PGE}_{2}$ [18]. Moreover, KFs produce less $\mathrm{PGE}_{2}$ in response to macrophage migration inhibitory factor (MIF) and have lower E prostanoid receptor 2 levels [19]. Given that $\mathrm{PGE}_{2}$ enhances MMP-1 expression [20], the reduced $\mathrm{PGE}_{2}$ levels in keloids may be responsible for the decreased MMP-1 production by keloids and their subsequent accumulation of extracellular matrix (ECM). Another lipid mediator, called cyclopentenone prostaglandin 15-deoxy- $\Delta^{12,14}$-prostaglandin $\mathrm{J}_{2}\left(15 \mathrm{~d}-\mathrm{PGJ}_{2}\right)$, also appears to have both pro-inflammatory and antiinflammatory activities. These activities are dependent on its concentration: at low concentrations, it enhances eotaxin-induced chemotaxis in eosinophils; but at high concentrations, it inhibits eosinophil survival by inducing apoptosis [21]. To our knowledge, little is known about the roles played in keloids by other products of AA metabolism, such as LT, PGI, and TX.

Given that AA has pro-inflammatory effects and EPA and its downstream product, DHA, can inhibit the production of inflammatory cytokines, such as IL-6 or TNF- $\alpha$ [22-24], Louw postulated that keloids may be treated by modulating the membrane FA composition, namely to reduce AA levels by supplementing the diet with GLA, DGLA, and EPA; it was proposed that this would restore the levels of AA precursors and inflammatory competitors and reduce the excessive release of AA [10]. This notion was supported by the fact that snake oil, which has high levels of LA, inhibits the growth of human KFs in vitro [25]. Moreover, this traditional African medicine, when consumed, both prevents and treats keloids [10].

Given that keloids, as fibroproliferative disorders, are to some extent the product of an imbalance between pro-and anti-inflammatory processes, attention should be paid to the "good" lipid mediators, namely, those that resolve and antagonize inflammation and even show antifibrotic effects, with the help of 5-LOX and 15-LOX. These "good" mediators include the LXs, protectin Ds (PDs), and resolvins (Rvs). Of these, the AA- and EPAderived LXs and the aspirin-triggered LXs (ATLs) have endogenous anti-inflammatory, proresolving and anti- 


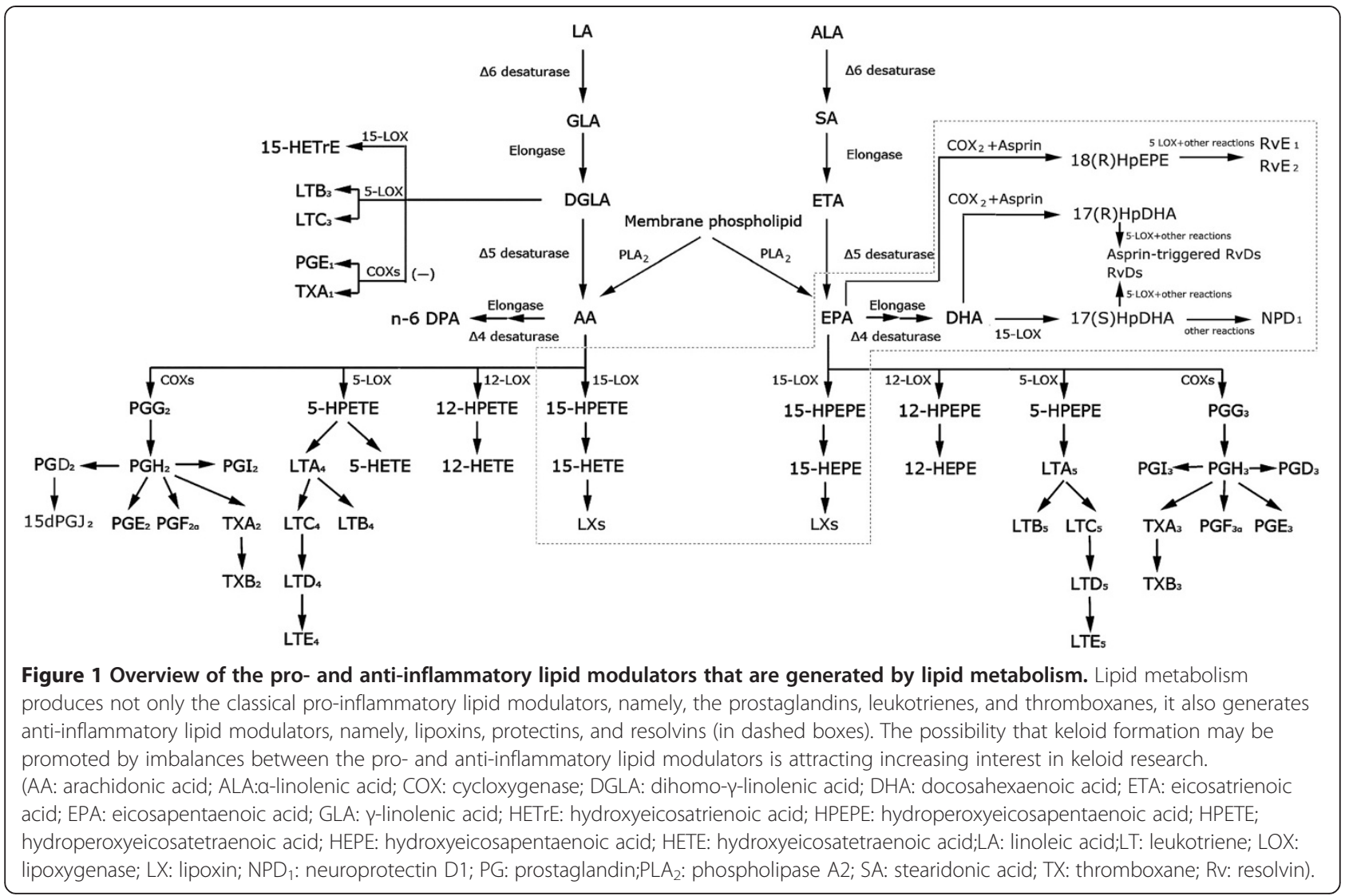

fibrotic functions [26,27]. Supporting evidence comes from studies on experimental dermal fibrosis: in this model, 15-LOX and its LX metabolites play a prominent anti-fibrotic role [28]. $\mathrm{LXA}_{4}$ and ATLa also attenuate experimental renal [29] and pulmonary [27] fibrosis, respectively. Similarly, the $\mathrm{Rv}$ and $\mathrm{PD}$ lipid mediators possess potent local anti-inflammatory and resolution properties [30]. However, although they can inhibit fibrosis in organs such as the kidney [31], little is known about their influence in skin and keloids. The mechanisms that cause switching between the lipid mediator classes, namely, from the generation of pro-inflammatory PGs and LTs to the production of antiinflammatory LXs, PDs, and Rvs, also remain to be clarified. This information will help to identify the pathways that can be targeted to inhibit or prevent keloids.

\section{Relationship between lipid-derived secondary messengers and keloids}

Lipids are not only constituents of the skin and can act as pro- and anti- inflammatory factors, they are also the source of secondary messengers that can influence the cellular events that are responsible for the development of keloids. These secondary messengers include diacylglycerol (DAG), ceramide and AA. In terms of keloid development, the most significant of these molecules is
DAG. DAG binds to the cysteine-rich domains of protein kinase $C$ (PKC) [32] and high levels of DAG correlate with persistent activation of PKC [33], which has been shown to contribute to the proliferation of fibroblasts in keloids [10]. Another important molecule in keloid development is ceramide, whose structure is similar to that of DAG. Ceramide is a lipid secondary messenger that is derived from cell membrane sphingomyelin and influences apoptosis pathways that are induced by stimuli such as Fas antigen [34,35]. Thus, ceramide mediates extracellular signals. Compared to normal dermal fibroblasts, KFs appear to be resistant to ceramide-induced apoptosis because they over-express insulin-like growth factor I (IGF-I) receptor [36]. It may also be that Fas-mediated signals are not transduced to ceramide in keloids and ceramide is not activated in anti-Fas-stimulated KFs [37]. With regard to AA, although it produces inflammatory factors and has long been known to participate in messenger systems that involve kinases, such as PKC, PKA, and mitogen-activated protein kinase (MAPK) [38], whether it can serve in KFs as secondary messengers remains unclear. Moreover, little seems to be known about the keloid-forming roles of other possible lipid-derived second messengers, such as phosphatidic acid (PA), lysophosphatidic acid (LPA), and inositol-1,4,5-triphosphate $\left(\mathrm{IP}_{3}\right)$. 


\section{Interactions between lipids and mechanotransduction in keloids}

Lipids are essential components of the membrane, which is the interface between the extracellular and intracellular compartments. This fact, together with the fact that lipids and their endogenous metabolites provides proand anti-inflammatory factors and secondary messengers, strongly supports the notion that lipids may influence the intracellular signaling pathways in keloids. Keloids are fibroproliferative skin disorders that appear increasingly to have a close relationship with local mechanical forces, as indicated by their preferred distribution in high-tension areas, their responsiveness to tension-reduction therapy, and their high levels of mechanotransduction pathway signaling [14,39]. Mechanotransduction is the process by which physical forces are converted into biochemical signals that are then integrated into cellular responses [40,41]. In keloids, the mechanotransduction pathways may involve the transforming growth factor- $\beta$ (TGF- $\beta$ )/Smad, MAPK, integrin, RhoA/ROCK, Wnt/ $\beta$-catenin, and TNF- $\alpha /$ nuclear factor kappa-light-chain-enhancer of activated B cells (NF-kB) pathways [42]. Therefore, research into possible intersections between these mechanotransduction pathways and lipids is likely to identify pathways and potential molecules that could be targeted by anti-keloid therapeutic approaches.

Several lines of research indicated the potential interactions between lipids and mechanotransduction. Lipid rafts are specialized plasma membrane microdomains enriched in cholesterol and sphingolipids, which may serve as signaling compartments $[43,44]$. As one type of lipid raft, caveolae are flask-shaped plasma membrane invaginations, whose major protein constituent is caveolin-1 [44]. Caveolae have already been proven to be important mechanotransduction sites in osteoblasts [45] and endothelial cells [46]. In endothelial cells, cholesterol depletion prevents the shear-induced activation of MAPKs [46] and caveolin-1 phosphorylation [47], and shear stress causes the phosphorylation of caveolin-1 and its recruitment to integrin sites [48]. Moreover, the $\beta 1$ integrin-mediated mechanotransduction in endothelial cells is mediated by caveolae domains [47]. In osteoblasts, disruption of cholesterol-rich plasma membrane compartments significantly reduces hydrostatic pressure- and shear-induced mechanotransduction. Moreover, restoration of plasma membrane rafts causes osteoblasts to regain their mechanotranducing properties [45]. The relationship between lipid rafts/caveolae and mechanotransduction in keloids is just being recognized. Evidence for this is that KFs have markedly decreased caveolin-1 expression and that treatment of KFs with caveolin-1 cell-permeable peptide (cav-1p) inhibited the TGF- $\beta 1$-induced up-regulation of collagen type I, fibronectin, and $\alpha$-SMA mRNA and protein expression. Moreover, the inhibitory effect of cav-1p on KF fibronectin production could be blocked by inhibiting the ERK1/2, but not the p38 and JNK pathways [49]. Further research is needed to clarify the relationship between lipids and the active mechanotransduction in keloids.

Lipids and their metabolites may also interact and shape the mechanotransduction pathways in keloids. These lipid molecules could be potential targets for pharmaceutical intervention that aims to prevent or treat keloids. There is evidence that the lipid metabolite $\mathrm{PGE}_{2}$ can inhibit keloid formation as follows. First, in KFs, the IL-1 $\beta$-induced up-regulation of COX-2 expression is impaired, resulting in a diminished stimulation of $\mathrm{PGE}_{2}$ secretion by IL- $1 \beta$. Second, the treatment of KFs with $\mathrm{PGE}_{2}$ partially reverses the ability of TGF- $\beta 1$ to upregulate the production of collagen types $\mathrm{I}$ and III. Third, $\mathrm{PGE}_{2}$ inhibits KF cell migration and contraction. Thus, $\mathrm{PGE}_{2}$ has both anti-fibroplastic and antiinflammatory effects on KFs [50]. Vitamin D may also be therapeutic for keloids, as shown by the fact that 1,25-dihydroxyvitamin D3 inhibits the TGF- $\beta 1$-induced secretion by KFs of matrix proteins such as collagen I, fibronectin, and $\alpha$-SMA [51]. Moreover, there is evidence that the TGF- $\beta$ and vitamin D signaling pathways may converge on SMAD [52], or at least cross-talk via the binding of vitamin D receptor and Smad3 proteins to their cognate DNA recognition elements [53]. The alkylphospholipid analogue hexadecylphosphocholine (HePC) could also be useful as a therapy because it can inhibit the proliferation of KFs; notably, the enhanced reorganization of collagen $\mathrm{I}$ in $\mathrm{KF}$ that is induced by $\mathrm{HePC}$ relates to the up-regulation of the $\alpha 2$-integrin chain [54].

\section{Conclusion}

In summary, in keloids, lipids not only serve as indispensable skin components, they also actively participate in the chronic inflammation processes that drive the development and progression of keloids and are typically manifested at the edge of keloid skin. Supporting this is that the levels of the metabolic products of the AA and EPA cascades are changed in keloids relative to normal skin. It is also likely that there is an imbalance between the pro-inflammatory PGs and LTs and the antiinflammatory LXs, PDs, and Rvs within these cascades that promotes inflammation. Lipids also serve as reservoirs of secondary messengers such as DAG and AA that contribute to fibroblast proliferation. Finally, there is evidence that mechanotransduction (an important etiological factor in keloid development) occurs in the lipid rafts and caveolae of the plasma membrane. Moreover, there are interactions between lipids and 
mechanotransduction pathway molecules, such as $\mathrm{PGE}_{2}$, Smads, and integrin. Thus, lipid molecules and their metabolic products may be potential pharmaceutical targets in interventions that aim to prevent, reduce, or even reverse keloid formation and/or progression.

\section{Abbreviations}

AA: Arachidonic acid; ALA: a-linolenic acid; ATLs: Aspirin-triggered lipoxins; COX: Cycloxygenase; DAG: Diacylglycerol; DGLA: Dihomo-y-linolenic acid; DHA: Docosahexaenoic acid; ECM: Extracellular matrix; EPA: Eicosapentaenoic acid; ETA: Eicosatrienoic acid; GLA: -linolenic acid; HePC: Hexadecylphosphocholine; HEPE: Hydroxyeicosapentaenoic acid; HETE: Hydroxyeicosatetraenoic acid; HPEPE: Hydroperoxyeicosapentaenoic acid; HETrE: Hydroxyeicosatrienoic acid; HPETE: Hydroperoxyeicosatetraenoic acid; IGF: Insulin-like growth factor; L: Interleukin; IP: Inositol-1,4,5triphosphate; LA: Linoleic acid; LOX: Lipoxygenase; LPA: Lysophosphatidic acid; LT: Leukotriene; LX: Lipoxin; MAPK: Mitogen-activated protein kinase; NFKB: Nuclear factor kappa-light-chain-enhancer of activated B cells; NPD 1 : Neuroprotectin D1; PA: Phosphatidic acid; PD: Protectin D; PG: Prostaglandin; PIP: Pl-4-phosphate; PIP: Phosphatidylinositol-4,5diphosphate; PKC: Protein kinase C; PLA : Phospholipase A2 PUFA: Polyunsaturated fatty acid; Rv: Resolvin; SA: Stearidonic acid; TGF- $\beta$ : Transforming growth factor- $\beta$; TX: Thromboxane

\section{Competing interests}

The authors declare that they have no competing interests. There are no non-financial competing interests.

\section{Authors' contributions}

All authors have made substantial contributions to this work. $\mathrm{CH}$ designed and drafted the manuscript after a literature research. RO guided the literature research and the drafting of the manuscript, and made the final corrections to the manuscript. Both authors have read and approved the final manuscript.

\section{Author details}

${ }^{1}$ Department of Plastic, Reconstructive and Aesthetic Surgery, Nippon Medical School, 1-1-5 Sendagi Bunkyo-ku, Tokyo 113-8603, Japan. ${ }^{2}$ Department of Plastic Surgery, Meitan General Hospital, Beijing, China.

Received: 1 March 2013 Accepted: 26 April 2013

Published: 1 May 2013

\section{References}

1. Lee JY, Yang CC, Chao SC, Wong TW: Histopathological differential diagnosis of keloid and hypertrophic scar. Am J Dermatopathol 2004 26:379-384.

2. Huang C, Akaishi S, Hyakusoku H, Ogawa R: Are keloid and hypertrophic scar different forms of the same disorder? A fibroproliferative skin disorder hypothesis based on keloid findings. Int Wound I 2012. doi:10.1111/j.1742-481X.2012.01118.x [Epub ahead of print].

3. Marneros AG, Norris JE, Watanabe S, Reichenberger E, Olsen BR: Genome scans provide evidence for keloid susceptibility loci on chromosomes 2q23 and 7p11. J Invest Dermatol 2004, 122:1126-1132.

4. Ogawa R: Keloid and hypertrophic scarring may result from a mechanoreceptor or mechanosensitive nociceptor disorder. Med Hypotheses 2008, 71:493-500.

5. Fong EP, Bay BH: Keloids - the sebum hypothesis revisited. Med Hypotheses 2002, 58:264-269.

6. Akaishi S, Ogawa R, Hyakusoku H: Keloid and hypertrophic scar: neurogenic inflammation hypotheses. Med Hypotheses 2008, 71:32-38.

7. Louw L, Engelbrecht AM, Cloete F, van der Westhuizen JP, Dumas L: Impairment in the fatty acid composition of keloids. Adv Exp Med Biol 1997, 400B:905-910.

8. Louw L: Keloids in rural black South Africans. Part 1: general overview and essential fatty acid hypotheses for keloid formation and prevention. Prostaglandins Leukot Essent Fatty Acids 2000, 63:237-245.

9. Louw L, Dannhauser A: Keloids in rural black South Africans. Part 2: dietary fatty acid intake and total phospholipid fatty acid profile in the blood of keloid patients. Prostaglandins Leukot Essent Fatty Acids 2000 63:247-253.

10. Louw L: Keloids in rural black South Africans. Part 3: a lipid model for the prevention and treatment of keloid formations. Prostaglandins Leukot Essent Fatty Acids 2000, 63:255-262.

11. Tachi M, Iwamori M: Mass spectrometric characterization of cholestero esters and wax esters in epidermis of fetal, adult and keloidal human skin. Exp Dermatol 2008, 17:318-323.

12. Pochi PE: The pathogenesis and treatment of acne. Annu Rev Med 1990, 41:187-198.

13. Kao JS, Fluhr JW, Man MQ, Fowler AJ, Hachem JP, Crumrine D, Ahn SK, Brown BE, Elias PM, Feingold KR: Short-term glucocorticoid treatment compromises both permeability barrier homeostasis and stratum corneum integrity: inhibition of epidermal lipid synthesis accounts for functional abnormalities. J Invest Dermatol 2003, 120:456-464.

14. Ogawa R, Okai K, Tokumura F, Mori K, Ohmori Y, Huang C, Hyakusoku H, Akaishi S: The relationship between skin stretching/contraction and pathologic scarring: the important role of mechanical forces in keloid generation. Wound Repair Regen 2012, 20:149-157.

15. Miles EA, Allen E, Calder PC: In vitro effects of eicosanoids derived from different 20-carbon Fattyacids on production of monocyte-derived cytokines in human whole blood cultures. Cytokine 2002, 20:215-223.

16. Levy BD, Clish CB, Schmidt B, Gronert K, Serhan CN: Lipid mediator class switching during acute inflammation: signals in resolution. Nat Immunol 2001, 2:612-619.

17. Serhan CN, Jain A, Marleau S, Clish C, Kantarci A, Behbehani B, Colgan SP, Stahl GL, Merched A, Petasis NA, Chan L, Van Dyke TE: Reduced inflammation and tissue damage in transgenic rabbits overexpressing 15-lipoxygenase and endogenous anti-inflammatory lipid mediators. J Immunol 2003, 171:6856-6865.

18. Yeh FL, Shen HD, Lin MW, Chang CY, Tai HY, Huang MH: Keloid-derived fibroblasts have a diminished capacity to produce prostaglandin E2. Burns 2006, 32:299-304.

19. Hayashi T, Nishihira J, Koyama Y, Sasaki S, Yamamoto Y: Decreased prostaglandin E2 production by inflammatory cytokine and lower expression of EP2 receptor result in increased collagen synthesis in keloid fibroblasts. J Invest Dermatol 2006, 126:990-997.

20. Sakaki H, Matsumiya T, Kusumi A, Imaizumi T, Satoh H, Yoshida H, Satoh K, Kimura H: Interleukin-1 beta induces matrix metalloproteinase-1 expression in cultured human gingival fibroblasts: role of cyclooxygenase-2 and prostaglandin E2. Oral Dis 2004, 10:87-93.

21. Ueki S, Kato H, Kobayashi Y, Ito W, Adachi T, Nagase H, Ohta K, Kayaba H, Chihara J: Anti- and proinflammatory effects of 15-deoxy-deltaprostaglandin J2(15d-PGJ2) on human eosinophil functions. Int Arch Allergy Immunol 2007, 143(Suppl 1):15-22.

22. Babcock TA, Novak T, Ong E, Jho DH, Helton WS, Espat NJ: Modulation of lipopolysaccharide-stimulated macrophage tumor necrosis factor-alpha production by omega- 3 fatty acid is associated with differential cyclooxygenase-2 protein expression and is independent of interleukin10. J Surg Res 2002, 107:135-139.

23. De Caterina R, Cybulsky MI, Clinton SK, Gimbrone MA Jr, Libby P: The omega-3 fatty acid docosahexaenoate reduces cytokine-induced expression of proatherogenic and proinflammatory proteins in human endothelial cells. Arterioscler Thromb 1994, 14:1829-1836.

24. Khalfoun B, Thibault F, Watier H, Bardos P, Lebranchu Y: Docosahexaenoic and eicosapentaenoic acids inhibit in vitro human endothelial cell production of interleukin-6. Adv Exp Med Biol 1997, 400B:589-597.

25. Datubo-Brown DD, Blight A: Inhibition of human fibroblast growth in vitro by a snake oil. Br J Plast Surg 1990, 43:183-186

26. Kieran NE, Maderna P, Godson C: Lipoxins: potential anti-inflammatory, proresolution, and antifibrotic mediators in renal disease. Kidney Int 2004, 65:1145-1154

27. Martins V, Valença SS, Farias-Filho FA, Molinaro R, Simões RL, Ferreira TP, e Silva PM, Hogaboam CM, Kunkel SL, Fierro IM, Canetti C, Benjamim CF: ATLa, an aspirin-triggered lipoxin A4 synthetic analog, prevents the inflammatory and fibrotic effects of bleomycin-induced pulmonary fibrosis. J Immunol 2009, 182:5374-5381.

28. Krönke G, Reich N, Scholtysek C, Akhmetshina A, Uderhardt S, Zerr P, Palumbo K, Lang V, Dees C, Distler O, Schett G, Distler JH: The 12/15-lipoxygenase pathway counteracts fibroblast activation and experimental fibrosis. Ann Rheum Dis 2012, 71:1081-1087. 
29. Börgeson E, Docherty NG, Murphy M, Rodgers K, Ryan A, O'Sullivan TP, Guiry PJ, Goldschmeding R, Higgins DF, Godson C: Lipoxin $\mathrm{A}_{4}$ and benzolipoxin $\mathrm{A}_{4}$ attenuate experimental renal fibrosis. FASEB J 2011, 25:2967-2979.

30. Serhan CN: Resolution phase of inflammation: novel endogenous antiinflammatory and proresolving lipid mediators and pathways. Annu Rev Immunol 2007, 25:101-137.

31. Qu X, Zhang X, Yao J, Song J, Nikolic-Paterson DJ, Li J: Resolvins E1 and D1 inhibit interstitial fibrosis in the obstructed kidney via inhibition of local fibroblast proliferation. J Pathol 2012. doi:10.1002/path.4050 [Epub ahead of print].

32. Zhang G, Kazanietz MG, Blumberg PM, Hurley JH: Crystal structure of the cys2 activator-binding domain of protein kinase $C$ delta in complex with phorbol ester. Cell 1995, 81:917-924.

33. Preiss J, Loomis CR, Bishop WR, Stein R, Niedel JE, Bell RM: Quantitative measurement of sn-1,2-diacylglycerols present in platelets, hepatocytes, and ras- and sis-transformed normal rat kidney cells. J Biol Chem 1986, 261:8597-8600.

34. Tepper CG, Jayadev S, Liu B, Bielawska A, Wolff R, Yonehara S, Hannun YA Seldin MF: Role for ceramide as an endogenous mediator of Fas-induced cytotoxicity. Proc Natl Acad Sci U S A 1995, 92:8443-8447.

35. Jayadev S, Liu B, Bielawska AE, Lee JY, Nazaire F, Pushkareva MYu, Obeid LM, Hannun YA: Role for ceramide in cell cycle arrest. J Biol Chem 1995. 270:2047-2052.

36. Ishihara H, Yoshimoto H, Fujioka M, Murakami R, Hirano A, Fujii T, Ohtsuru A, Namba H, Yamashita S: Keloid fibroblasts resist ceramide-induced apoptosis by overexpression of insulin-like growth factor I receptor. J Invest Dermatol 2000, 115:1065-1071.

37. Lu F, Gao J, Ogawa R, Hyakusoku H, Ou C: Fas-mediated apoptotic signal transduction in keloid and hypertrophic scar. Plast Reconstr Surg 2007, 119:1714-1721.

38. Sellmayer A, Danesch U, Weber PC: Modulation of the expression of early genes by polyunsaturated fatty acids. Prostaglandins Leukot Essent Fatty Acids 1997, 57:353-357.

39. Ogawa R, Akaishi S, Huang C, Dohi T, Aoki M, Omori Y, Koike S, Kobe K, Akimoto $\mathrm{M}$, Hyakusoku $\mathrm{H}$ : Clinical applications of basic research that shows reducing skin tension could prevent and treat abnormal scarring: the importance of fascial/subcutaneous tensile reduction sutures and flap surgery for keloid and hypertrophic scar reconstruction. J Nihon Med Sch 2011, 78:68-76.

40. Huang H, Kamm RD, Lee RT: Cell mechanics and mechanotransduction: pathways, probes, and physiology. Am J Physiol Cell Physiol 2004, 287:C1-C11.

41. Ingber DE: Mechanobiology and diseases of mechanotransduction. Ann Med 2003, 35:564-577.

42. Huang C, Ogawa R: Fibroproliferative disorders and their mechanobiology. Connect Tissue Res 2012, 53:187-196.

43. Simons K, Toomre D: Lipid rafts and signal transduction. Nat Rev Mol Cell Biol 2000, 1:31-39.

44. Allen JA, Halverson-Tamboli RA, Rasenick MM: Lipid raft microdomains and neurotransmitter signalling. Nat Rev Neurosci 2007, 8:128-140.

45. Ferraro JT, Daneshmand M, Bizios R, Rizzo V: Depletion of plasma membrane cholesterol dampens hydrostatic pressure and shear stress-induced mechanotransduction pathways in osteoblast cultures. Am J Physiol Cell Physiol 2004, 286:C831-C839.

46. Park H, Go YM, St John PL, Maland MC, Lisanti MP, Abrahamson DR, Jo H: Plasma membrane cholesterol is a key molecule in shear stressdependent activation of extracellular signal-regulated kinase. J Biol Chem 1998, 273:32304-32311.

47. Radel C, Carlile-Klusacek M, Rizzo V: Participation of caveolae in beta1 integrin-mediated mechanotransduction. Biochem Biophys Res Commun 2007, 358:626-631

48. Radel C, Rizzo V: Integrin mechanotransduction stimulates caveolin-1 phosphorylation and recruitment of Csk to mediate actin reorganization. Am J Physiol Heart Circ Physiol 2005, 288:H936-H945.

49. Zhang GY, Yu Q, Cheng T, Liao T, Nie CL, Wang AY, Zheng X, Xie XG, Albers $A E$, Gao WY: Role of caveolin-1 in the pathogenesis of tissue fibrosis by keloid-derived fibroblasts in vitro. Br J Dermatol 2011, 164:623-627.

50. Sandulache VC, Parekh A, Li-Korotky H, Dohar JE, Hebda PA: Prostaglandin E2 inhibition of keloid fibroblast migration, contraction, and transforming growth factor (TGF)-beta1-induced collagen synthesis. Wound Repair Regen 2007, 15:122-133.
51. Zhang GY, Cheng T, Luan Q, Liao T, Nie CL, Zheng X, Xie XG, Gao WY: Vitamin D: a novel therapeutic approach for keloid, an in vitro analysis. Br J Dermatol 2011, 164:729-737.

52. Yanagisawa J, Yanagi $Y$, Masuhiro $Y$, Suzawa M, Watanabe M, Kashiwagi $K$, Toriyabe T, Kawabata M, Miyazono K, Kato S: Convergence of transforming growth factor-beta and vitamin D signaling pathways on SMAD transcriptional coactivators. Science 1999, 283:1317-1321.

53. Subramaniam N, Leong GM, Cock TA, Flanagan JL, Fong C, Eisman JA, Kouzmenko AP: Cross-talk between 1,25-dihydroxyvitamin D3 and transforming growth factor-beta signaling requires binding of VDR and Smad3 proteins to their cognate DNA recognition elements. J Biol Chem 2001, 276:15741-15746.

54. Blume-Peytavi U, Geilen CC, Sommer C, Almond-Roesler B, Orfanos CE: The phospholipid analogue hexadecylphosphocholine (HePC) inhibits proliferation of keloid fibroblasts in vitro and modulates their fibronectin and integrin synthesis. Arch Dermatol Res 1997, 289:164-169.

doi:10.1186/1476-511X-12-60

Cite this article as: Huang and Ogawa: Roles of lipid metabolism in keloid development. Lipids in Health and Disease 2013 12:60.

\section{Submit your next manuscript to BioMed Central and take full advantage of:}

- Convenient online submission

- Thorough peer review

- No space constraints or color figure charges

- Immediate publication on acceptance

- Inclusion in PubMed, CAS, Scopus and Google Scholar

- Research which is freely available for redistribution

Submit your manuscript at www.biomedcentral.com/submit
C BioMed Central 